
\title{
R Resarach S Surare \\ The Effect of Psychological Factors on the Quality of Life in Iranian Lactating Women During the COVID-19 Pandemic: A Path Analysis
}

\section{Youseflu Samaneh}

Zanjan University of Medical Sciences

Shahideh Jahanian Sadatmahalleh ( $\sim$ Shahideh.Jahanian@modares.ac.ir)

Tarbiat Modares University Faculty of Medical Sciences

Jahangiri Nadia

ACECR

Azam Maleki

Zanjan University of Medical Sciences

Ashraf Moini

Tehran University of Medical Sciences School of Medicine

Research

Keywords: Quality of Life, Path Analysis, HADS, Breastfeeding, COVID-19

Posted Date: December 11th, 2020

DOl: https://doi.org/10.21203/rs.3.rs-125033/v1

License: (c) (i) This work is licensed under a Creative Commons Attribution 4.0 International License.

Read Full License 


\section{Abstract}

Background: breastfeeding women are susceptible to mental disorders particularly during outbreaks of infectious diseases such as COVID-19. In this study we develop a conceptual model considering the interrelated role of anxiety, depression, general health, breastfeeding, education, income, and corona disease anxiety on the QoL of breastfeeding women.

Method: In a descriptive-analytical study, 279 breastfeeding women were recruited from March to April 2020. Data were collected using a socio-demographic checklist, short Form of Health Survey (SF_12), Hospital Anxiety and Depression Scale (HADS), General Health Questionnaire (GHQ), and Corona Disease Anxiety Scale (CDAS).

Results: The overall goodness-of-fit statistics showed that the conceptual model was acceptable (GFI $=0.99$, RMSEA =0.06). Results showed that breast-feeding, GHQ, anxiety, depression, and education have a direct effect on women's QoL. We found significant total mediating effects of GH and breastfeeding and moderating effects of education and income on quality of life. Income had indirectly effect on QOL through the education.

Conclusion: Our results give support for a hypothetical model in which improvement in breastfeeding, anxiety, depression, general health, education, and income led to higher levels of QoL in breastfeeding women. It seems that the predictors proposed by this model are greatly important and should be considered when designing optimal psychological care interventions.

\section{Background}

Infectious disease disasters consist of outbreaks, epidemics and pandemics may result in high morbidity and mortality and may contribute to nearly $25-33 \%$ of global death rate (1). The 2019 novel coronavirus disease (COVID-19) is a new and serious infectious disease which was first detected in Wuhan, China, in December, 2019 and has been announced by WHO as the sixth global public health emergency $(1,2)$. Evidence suggest that individuals may suffer the wide and profound psychological impact during outbreaks of communicable diseases which can potentially have a mental breakdown varying from anxiety, depression and panic attacks to delirium, psychosis and even suicidality (3-5). These mental illness can be harmful to quality of life (6). Quality of life (QoL) as a multidimensional theoretical construct which covers all aspects of people's lives including physical health, psychological state and level of independence and social communication, is the principal concerns of health care providers and is the best indicator for assessing health status (7). On the other hand, breastfeeding is one of the most effective methods to guarantee child health and survival (8). Furthermore, to being a significant source of nutrition to the child, investigations demonstrate that breastfeeding is not just a meal at the breast but also has important and extensive effects on cognition, behavior, and mental health in children and mothers (9). There are researches showing a negative association between experience of breastfeeding and parent-reported antisocial and aggressive behavior in offspring in middle childhood (10). Numerous 
factors such as physiological and psychological factors are involved in the beginning and continuation of breastfeeding (11). Because of the importance of breastfeeding and considering the high prevalence of the COVID-19 disease and its different psychological impacts on human lives and the existence of limited studies on the psychological health of breastfeeding women during the COVID-19 pandemic, we decided to assess variations in the psychosocial functioning associated with COVID-19 and determine its influence on QoL in breastfeeding women in Iran using path analysis. In this study we develop a conceptual model considering the interrelated role of anxiety, depression, general health, breastfeeding, education, income, and corona disease anxiety on the QoL of breastfeeding women. Based on the above aims, this study proposes the following hypotheses (Hypotheses 1-4):

Hypothesis 1: A higher level of anxiety and depression will be associated with a lower level of general health, and also have a worse effect on the breastfeeding, and QoL.

Hypothesis 2: A higher level of CDAS will be associated with higher level of anxiety, depression, general health and have a worse effects on breastfeeding, and QoL.

Hypothesis 3: General health will be associated with CDAS, anxiety, depression, and have a negative on the breastfeeding, and QoL.

Hypothesis 4: A higher level of education, and income will be associated with anxiety, depression, general health, breastfeeding, and QoL.

\section{Methods}

\section{Design and Data Collection:}

This descriptive-analytical study was conducted between March to April 2020. The project used a nonprobability, convenience sample of 279 eligible breastfeeding women. The protocol was approved by the Institutional Review Board and Research Ethics Committee of Tarbiat Modares University (code:

R.MODARES.REC.1399.004)). The consent was obtained from participants in the study and they all made sure that their information will be kept private and confidential.

An electronic questionnaire (https://survey.porsline.ir/s/qahAeAY) was used to gather the data in order to maintain physical distance to prevent coronavirus disease. The designed questionnaires on the web were sent to breastfeeding women. The aim of the study, inclusion criteria and the way of responding to questions were mentioned on the first page.

Eligibility criteria required individuals to have infant under 6 month old; to be healthy, with no identifiable mental disorder or medical disease; have $\mathrm{BMK} 40$; and to be sufficiently literate to be able to understand and answer all questions; have no corticosteroid therapy, a history of chemotherapy, malignancies, organ transplants, HIV infection, cardiovascular disease, high blood pressure, diabetes, and respiratory illness.

\section{Measures:}




\section{Socio-demographic and obstetric check list}

At first, a checklist of socio-demographic and obstetric variables including women's age, gravidity, parity, number of live children, age at marriage, duration of marriage, previous mode of delivery, body mass index, residency, monthly income, level of educational, occupational status, type of pregnancy (planed or implanted pregnancy), contraception method, and type and number of breastfeeding per day (exclusive breastfeeding, bottle feeding, or combination of two methods).

\section{The General Health Questionnaire (GHQ)}

The General Health Questionnaire (GHQ) evaluate four dimensions of physical symptoms (1-7), anxiety and insomnia (8-14), social function (15-21), and depression (28-22) (12). Higher scores represent worse general health. The validity and reliability of this questionnaire which was first developed by Goldberg (1978) have been also confirmed in Iran $(13,14)$.

\section{Hospital Anxiety and Depression Scale (HADS)}

Hospital Anxiety and Depression Scale (HADS) is a widely used instrument to measure the severity of depression and anxiety consisting of 14 questions composed of two subscales of anxiety (HADS-A) and depression (HADS-D). Each question is ranked on a four point Likert scale, into three levels of normal range (total score $<$ ), slight changes (score of 8-10) and high levels of anxiety and depression (total score $>11)$. In Iran, the validity and reliability of this questionnaire were investigated by Montazeri et al. (15).

Corona Disease Anxiety Scale (CDAS) Preliminary validation of the Corona Disease Anxiety Scale (CDAS) in Iran has been prepared by Alipour et al. to measure anxiety caused by coronavirus outbreaks. This questionnaire has 18 items to measure psychological symptoms (Items 1 to 9 ) and physical symptoms (items 10 to 18). This tool is rated on a 4-point Likert scale ranging from a score of 0 to 54 as the lowest and highest scores are given by the respondents. High scores on this questionnaire is a sign of higher level of anxiety in individuals. The validity and reliability of this questionnaire are well documented (16).

\section{Quality of life}

Quality of life assessment was performed using the short form 12 questionnaire (SF12) which is composed of eight subscales of physical performance, physical role, social role, emotional role, body pain, general health, vitality, and mental health. Questions 1, 8, 9, and 10 were negative, so their scoring was reversed. Each item scored from 1 to 6 , and total scores computed from 0 to 100 . A higher score indicates the best situations. The questions were in the form of a Likert ranges from score 1 to 6 . In Iran, the psychometric properties of this questionnaire was confirmed (17).

\section{Data analysis:}


Data were analyzed using a software package used for statistical analysis (SPSS version 22, SPSS, Inc.) and linear structural relations (LISREL; version 8.8). Multiple correlation coefficients as a test which assesses the effects of independent variables on the dependent variable, were used to determine the relationship between QoL variables, anxiety and depression, general health, education, income, breastfeeding and corona disease anxiety.

Path analysis has been applied to generate the specified pattern of causal relationships between variables. Path analysis determines the type of relationship between predicted variables and responsible variables. In this study, a conceptual model of path analysis to determine the simultaneous relationship Between QoL, anxiety and depression, general health, breastfeeding, and corona disease anxiety was a good fit to the data.

After analyzing the fit of the data and finding the best fit, the acceptable model was determined. For evaluation of the model fitness, Root means a square error of approximation (RMSEA), Adjusted Goodness of Fit Index (AGFI), Confirmatory Factor Analytic (CFI), and Chi-square/df were applied. RMSEA values less than 0.08 , Chi-square/df lower than 3 , AGFI more than 0.9 , and CFI more than 0.95 are the characteristics of a good fitting model.

\section{Results}

Table 1 describes the demographic characteristics of participants. The mean age of participants and duration of their marriage were $32.37 \pm 3.60$ and $5 \pm 3.91$ years, respectively. The majority of participants $(60.57 \%)$ had exclusive breastfeeding. In terms of educational level, $73.12 \%$ of participants had academic level education. 
Table 1

Demographic Characteristics of Breastfeeding Women $(\mathrm{N}=279)$.

\begin{tabular}{|c|c|}
\hline \multicolumn{2}{|l|}{ Characteristic } \\
\hline Age (years) ${ }^{*}$ & $32.37 \pm 3.60$ \\
\hline Parity * & $1.04 \pm 0.53$ \\
\hline Duration of marriage (years) $^{*}$ & $5 \pm 3.91$ \\
\hline \multicolumn{2}{|l|}{ Type of breastfeeding ** } \\
\hline Bottle feeding & $38(13.62)$ \\
\hline Combination & $72(25.81)$ \\
\hline Exclusive breastfeeding & $169(60.57)$ \\
\hline \multicolumn{2}{|l|}{ Income (Taman) ** } \\
\hline Less than 1 million & 34 (12.19) \\
\hline Between 1-3 million & $82(29.39)$ \\
\hline Between 3-5 million & $121(43.37)$ \\
\hline More than 5 million & $42(15.05)$ \\
\hline \multicolumn{2}{|l|}{ Education $^{\star *}$} \\
\hline High school & $75(26.88)$ \\
\hline University & $204(73.12)$ \\
\hline QoL $^{*}$ & $71.66 \pm 12.3$ \\
\hline
\end{tabular}

The overall mean score of QoL was $71.66 \pm 12.3$. Table 2 shows the correlation (bivariate analysis) between all variables submitted to the path model. There was a negative significant correlation between QoLwith anxiety $(r=-0.52, p<0.001)$, depression $(r=-0.45, p<0.001), G H(r=-0.47, p<0.001)$, CDAS $(r=-0.38$, $p<0.001)$. 
Table 2

Correlations between $\mathrm{QOL}, \mathrm{CDAS}, \mathrm{GHQ}$, anxiety, and depression.

\begin{tabular}{|c|c|c|c|c|}
\hline & 1 & 2 & 3 & 4 \\
\hline 1. QOL & - & - & - & - \\
\hline 2. Anxiety & $-0.52^{\star \star \star}$ & - & - & - \\
\hline 3. Depression & $-0.45^{\star \star \star}$ & $0.67^{\star \star}$ & - & - \\
\hline 4. $\mathrm{GH}$ & $-0.47^{\star \star \star}$ & $0.69^{\star \star \star}$ & $0.63^{\star \star \star}$ & - \\
\hline 7. CDAS & $-0.38^{\star \star \star}$ & $0.42^{\star \star \star}$ & $0.27^{\star \star \star}$ & $0.32^{\star \star \star}$ \\
\hline
\end{tabular}

Path diagram for the predictors of quality of life had good fitness indices (P-value $=0.02$; chi2 $=22.35 ; D F$ $=11 ;$ chi $2 / \mathrm{df}=2.03 ;$ RMSEA $=0.06 ; \mathrm{CFI}=0.99 ; \mathrm{AGFI}=0.94)($ Table 3$)$.

Table 3

The goodness of Fit Indices for the Models.

\begin{tabular}{|c|c|c|c|c|c|c|c|}
\hline & $\mathrm{CFI}$ & AGFIOL & RMSEA IIU & Chi-square & df & Chi-square/df吅 & P-value \\
\hline $\begin{array}{l}\text { Path } \\
N=279\end{array}$ & 0.99 & 0.94 & 0.06 & 22.35 & 11 & 2.03 & 0.02 \\
\hline
\end{tabular}

Quality of life and moderators: Table 4 and Fig. 1 show the direct, indirect, and total effects of psychological variables on the QoL of lactating women. Results showed that breast-feeding $(\beta=0.29)$, $\mathrm{GH}(\beta=-0.21)$, anxiety $(\beta=-0.64)$, depression $(\beta=-0.34)$, and education $(\beta=2.50)$ have a direct effect on women's QoL. We found moderating effects of education and income on quality of life Income had indirectly effect on QOL through the education. 
Table 4

Path coefficients for breast-feeding, GH, anxiety, depression, CDAS, income, education, and QOL of lactating women during pandemic of corona virus.

\begin{tabular}{|c|c|c|c|c|c|}
\hline & & Direct effect & Indirect effect & Total effect & T-value \\
\hline \multirow[t]{8}{*}{ QOL } & Breast-feeding & 0.29 & - & 0.29 & 2.45 \\
\hline & $\mathrm{GH}$ & -0.21 & -0.02 & -0.23 & -3.40 \\
\hline & Anxiety & -0.64 & -0.66 & -1.30 & -9.54 \\
\hline & Depression & -0.34 & -0.20 & -0.54 & -2.79 \\
\hline & Education & 2.50 & 0.76 & 3.26 & 6.63 \\
\hline & CDAS & - & -0.35 & -0.35 & -7.53 \\
\hline & Income & - & 0.94 & 0.94 & 3.17 \\
\hline & CDAS & -0.13 & -0.04 & -0.17 & -5.87 \\
\hline \multirow[t]{3}{*}{ Breast-feeding } & Depression & - & -0.05 & -0.05 & -1.95 \\
\hline & $\mathrm{GH}$ & -0.05 & - & -0.05 & -2.11 \\
\hline & Anxiety & - & -0.10 & -0.10 & -2.09 \\
\hline \multirow[t]{6}{*}{$\mathrm{GH}$} & CDAS & - & 0.48 & 0.48 & 8.62 \\
\hline & Depression & 0.91 & - & 0.91 & 5.20 \\
\hline & Anxiety & 1.47 & 0.55 & 2.02 & 16.54 \\
\hline & Income & - & -0.34 & -0.34 & -2.44 \\
\hline & Education & - & -1.18 & -1.18 & -3.31 \\
\hline & anxiety & 0.60 & - & 0.60 & 15.05 \\
\hline \multirow[t]{3}{*}{ Depression } & CDAS & - & 0.14 & 0.14 & 8.39 \\
\hline & income & - & -0.10 & -0.10 & -2.43 \\
\hline & Education & - & -0.35 & -0.35 & -3.29 \\
\hline \multirow[t]{3}{*}{ Anxiety } & CDAS & 0.24 & - & 0.24 & 10.10 \\
\hline & Income & - & -0.17 & -0.17 & -2.46 \\
\hline & Education & -0.59 & - & -0.59 & -3.37 \\
\hline
\end{tabular}


Breast-feeding as one of the main predictors of QoL was affected by $\mathrm{GH}$, anxiety, depression, and CDAS. CDAS only with indirect effect $(\beta=-0.35)$ through anxiety, depression, $G H Q$, and breast feeding could be predicted QoL. More ever, GH was associated with anxiety $(\beta=2.02)$, depression $(\beta=0.91), \operatorname{CDAS}(\beta=$ 0.48 ), income $(\beta=-0.34)$, and education $(\beta=-1.18)$. We found significant total mediating effects of $\mathrm{GH}$ and breastfeeding on QoL.

\section{Discussion}

In this current study, we used pathway analysis to establish a relationship between theoretical frameworks around related factors of QoL in Iranian lactating women during the pandemic of coronavirus. Our findings demonstrated that breastfeeding, anxiety, depression, general health, income, education, and corona related anxiety was significant along the pathway of predictors of QoL in breastfeeding women.

The results of path analysis showed that a higher level of anxiety and depression led to a lower level of QOL in the form of a direct pathway. Moreover, anxiety with indirect effects through depression, general health, and breast-feeding can affect women's QoL.

The result of the Ceulemans et al. study reported a high prevalence of anxiety and depression in pregnant and breastfeeding women during the COVID-19 pandemic (18). Women during the postpartum period, already vulnerable owing to mood and psychological disorders. The outbreak of COVID-19, fear of infection, and its associated quarantine measures have many negative impacts on the psychological wellbeing of breastfeeding women (19). On the other hand, public health measures can interfere with the psychosocial needs of the postpartum period (19). For example, for many new mothers, recommended isolation and social distancing has inevitably interfered with staff one-on-one support, and interaction between women admitted to the same ward. Nanjundaswamy et al. study showed that higher level of anxiety-related corona among pregnant, and breastfeeding mothers was related with concern about hospital visit, social media messages, the safety of the infant, methods of protection, and contracting the infection (20). Moreover, a recent study reported that during the covid-19 pandemic, variables such as marital satisfaction, the level of husband education, spouse's support, and the number of pregnancies were the main predictors of anxiety in pregnant women (21).

Maternal mental health and its effects on breastfeeding are considered as a bellwether during the pandemic of COVID-19. Our results revealed that anxiety, depression, and CDAS have a negative impact on breastfeeding. The result of one systematic review shows that postpartum anxiety has negative impacts on breastfeeding initiation, duration, and exclusivity (22). Transmission of covid-19 as a respiratory virus via breast milk is not common even for mothers who tested positive (23). The properties of breast milk including anti-infective factors, as well as transferring of immunological competence and memory can protect the infant against infection disease (23). Mother anxiety has an adverse physiological effect on breastfeeding due to maternal anxiety and stress can interfere with oxytocin releasing and milk ejection reflex. On the other hand anxiety with diminishing self-esteem, and mother- 
infant interactions have negative effects on breastfeeding (22). Worsening of mother-infant bonding one month after childbirth was reported after the COVID-19 pandemic (24). Hull et al.'s study indicated that during the pandemic of COVID - 19, mothers who had not initiated or had stopped breastfeeding would like to start/restart breastfeeding due to concern regarding infant formula availability (25). The results of Zubaran et al.'s study was shown a positive correlation between breastfeeding and QoL, but in their study, no significant relation was seen between the level of education, socioeconomic status, and breastfeeding efficacy (26).

In our study, anxiety is considered the main risk factor for depression. Besides, some variables such as education, income, and CDAS were predictors of depression. More ever, depression, and anxiety have negative impact on general health, and breastfeeding. One study reported worsening of postpartum depression in pregnant and breastfeeding women during the pandemic of COVID-19 (18). The result of Wu et al.'s study on pregnant women during the pandemic of covid-19 indicated that depression was related to lower level of education, income, worked part-time, poorer family support, lower level of physical exercise, per capita living area of less than $20 \mathrm{~m}^{2}$ (27). Ma et al. study reported that in the general population depression was related to low QoL (28).

Our finding suggested the level of education as the most important factor in the QoL. More ever education with indirect effects through anxiety, depression, and general health can change QoL.

This results is in line with previous study (10). Therefore empowering, and promoting the socio-economic of breastfeeding mothers can improve the level of anxiety, general health, depression, as well as QoL.

To our knowledge several studies have evaluated that anxiety, depression, breastfeeding, and general health contribute to the QOL of breastfeeding mothers independently. The combination of the evaluated domains is the main strength of the present study. The use of validated questionnaires, and utilizing a developed conceptual model are other strengths of the current study. More ever, it is the first study that evaluated influential factors on breastfeeding, and QoL of breastfeeding women during pandemic of covid-19 using path analysis.

Despite the strengths of the current study, our finding have some limitations. First, regarding the crosssectional study design, causal relationships among variables cannot be drawn. On the other hand, we don't have information about the quality of life and mental health of women before participating in the study. Second, we did not evaluate the influence of other variables (such as, social support, violence, selfesteem, and cultural issues, etc.) that can effect on women's QoL. It has been offered, that future studies consider these issues.

\section{Conclusion}

The results presented in this article give support for a hypothetical model in which improvement in breastfeeding, anxiety, depression, general health, education, and income led to higher levels of QoL in 
breastfeeding women. It seems that the predictors proposed by this model are greatly important and should be considered when designing optimal psychological care interventions.

\section{Abbreviations}

QoL: Quality of Life

HADS: Hospital Anxiety and Depression Scale

GH: General Health;

CDAS: Corona Disease Anxiety Scale

RMSEA: Root mean square error of approximation

AGFI: Adjusted Goodness of Fit Index

CFI: Confirmatory Factor Analytic

DF: Degree of Freedom

\section{Declarations}

\section{Ethics approval and consent to participate}

The study was approved by the Ethics Committee of Tarbiat Modares University of Medical Sciences (IR.MODARES.REC.1399.004). All procedures were in accordance with the ethical standards of the Regional research committee and with the Declaration of Helsinki 1964 and its later amendments. After explaining the study's purposes, written consent assent were collected from all participants and women were informed that their participation were voluntary, con

\section{Consent for publication}

Not applicable.

\section{Availability of data and materials}

The data sets used and analyzed during the current study are available from the corresponding author on reasonable request

\section{Competing interests}

The authors declare no conflict of interest.

\section{Funding}


None.

\section{Authors' contributions}

SH.J and A.M, S.Y contributed to the conception and design of the study; SY, N.J and SH.J did the literature search; S.Y, and AZ.M performed the statistical analysis; SY, SHJ, and N.J wrote the first draft of the manuscript. All authors contributed to manuscript revision, read, and approved the submitted version.

\section{Acknowledgments}

This study was carried out with the kind collaboration of the participants. This study is a part of research work done in Tarbiat Modares University, Tehran, Iran. There were no conflicts of interest.

\section{References}

1. Zhu N, Zhang D, Wang W, Li X, Yang B, Song J, et al. A novel coronavirus from patients with pneumonia in China, 2019. New England Journal of Medicine. 2020.

2. World Health O. Mental health and psychosocial considerations during the COVID-19 outbreak, 18 March 2020. Geneva: World Health Organization; 2020 2020. Contract No.: WHO/2019nCoV/MentalHealth/2020.1.

3. Müller N. Infectious Diseases and Mental Health. Comorbidity of Mental and Physical Disorders. 2014:99.

4. Organization WH. Mental health and psychosocial considerations during the COVID-19 outbreak, 18 March 2020. World Health Organization; 2020.

5. Ho CS, Chee CY, Ho RC. Mental health strategies to combat the psychological impact of COVID-19 beyond paranoia and panic. Ann Acad Med Singapore. 2020;49(1):1-3.

6. Chiang YS, Chang YC, Liu YP, Tzeng WC. Quality of life in patients with comorbid serious mental illness and chronic diseases: A structural equation model. Journal of Advanced Nursing. 2020.

7. Fayers PM, Machin D. Quality of life: the assessment, analysis and interpretation of patient-reported outcomes: John Wiley \& Sons; 2013.

8. Krol KM, Grossmann T. Psychological effects of breastfeeding on children and mothers. Bundesgesundheitsblatt-Gesundheitsforschung-Gesundheitsschutz. 2018;61(8):977-85.

9. Shelton KH, Collishaw S, Rice FJ, Harold GT, Thapar A. Using a genetically informative design to examine the relationship between breastfeeding and childhood conduct problems. European child \& adolescent psychiatry. 2011;20(11-12):571-9.

10. Mirghafourvand M, Kamalifard M, Ranjbar F, Gordani N. Relationship of breastfeeding self-efficacy with quality of life in Iranian breastfeeding mothers. The Journal of Maternal-Fetal \& Neonatal Medicine. 2018;31(20):2721-8.

11. Gila-Díaz A, Carrillo GH, López de Pablo ÁL, Arribas SM, Ramiro-Cortijo D. Association between Maternal Postpartum Depression, Stress, Optimism, and Breastfeeding Pattern in the First Six 
Months. International Journal of Environmental Research and Public Health. 2020;17(19):7153.

12. Sterling M. General health questionnaire-28 (GHQ-28). Journal of physiotherapy. 2011;57(4):259.

13. Malakouti SK, Fatollahi P, Mirabzadeh A, Zandi T. Reliability, validity and factor structure of the GHQ28 used among elderly Iranians. International Psychogeriatrics. 2007;19(4):623-34.

14. Taghavi S. Validity and reliability of the general health questionnaire (GHQ-28) in college students of Shiraz University. Journal of psychology. 2002;5(4):381-98.

15. Montazeri A, Vahdaninia M, Ebrahimi M, Jarvandi S. The Hospital Anxiety and Depression Scale (HADS): translation and validation study of the Iranian version. Health and quality of life outcomes. 2003;1(1):14.

16. ALIPOUR A, Ghadami A, ALIPOUR Z, ABDOLLAHZADEH H. Preliminary validation of the Corona Disease Anxiety Scale (CDAS) in the Iranian sample. 2020.

17. Montazeri A, Vahdaninia M, Mousavi SJ, Omidvari S. The Iranian version of 12-item Short Form Health Survey (SF-12): factor structure, internal consistency and construct validity. BMC public health. 2009;9(1):341.

18. Ceulemans M, Hompes T, Foulon V. Mental health status of pregnant and breastfeeding women during the COVID-19 pandemic: A call for action. International Journal of Gynecology \& Obstetrics. 2020;151(1):146-7.

19. Hermann A, Fitelson EM, Bergink V. Meeting Maternal Mental Health Needs During the COVID-19 Pandemic. JAMA Psychiatry. 2020.

20. Nanjundaswamy MH, Shiva L, Desai G, Ganjekar S, Kishore T, Ram U, et al. COVID-19-related anxiety and concerns expressed by pregnant and postpartum women-a survey among obstetricians. Archives of Women's Mental Health. 2020.

21. Effati-Daryani F, Zarei S, Mohammadi A, Hemmati E, Ghasemi Yngyknd S, Mirghafourvand M. Depression, stress, anxiety and their predictors in Iranian pregnant women during the outbreak of COVID-19. BMC Psychology. 2020;8(1):99.

22. Hoff CE, Movva N, Rosen Vollmar AK, Pérez-Escamilla R. Impact of maternal anxiety on breastfeeding outcomes: a systematic review. Advances in Nutrition. 2019;10(5):816-26.

23. Lubbe W, Botha E, Niela-Vilen H, Reimers P. Breastfeeding during the COVID-19 pandemic - a literature review for clinical practice. International Breastfeeding Journal. 2020;15(1):82.

24. Suzuki S. Psychological status of postpartum women under the COVID-19 pandemic in Japan. The Journal of Maternal-Fetal \& Neonatal Medicine. 2020:1-3.

25. Hull N, Kam RL, Gribble KD. Providing breastfeeding support during the COVID-19 pandemic: Concerns of mothers who contacted the Australian Breastfeeding Association. medRxiv. 2020:2020.07.18.20152256.

26. The Correlation Between Breastfeeding and Maternal Quality of Life in Southern Brazil. Breastfeeding Medicine. 2011;6(1):25-30. 
27. Wu Y, Zhang C, Liu H, Duan C, Li C, Fan J, et al. Perinatal depressive and anxiety symptoms of pregnant women during the coronavirus disease 2019 outbreak in China. Am J Obstet Gynecol. 2020;223(2):240.e1-.e9.

28. Ma Y-F, Li W, Deng H-B, Wang L, Wang Y, Wang P-H, et al. Prevalence of depression and its association with quality of life in clinically stable patients with COVID-19. Journal of Affective Disorders. 2020;275:145-8.

Figures

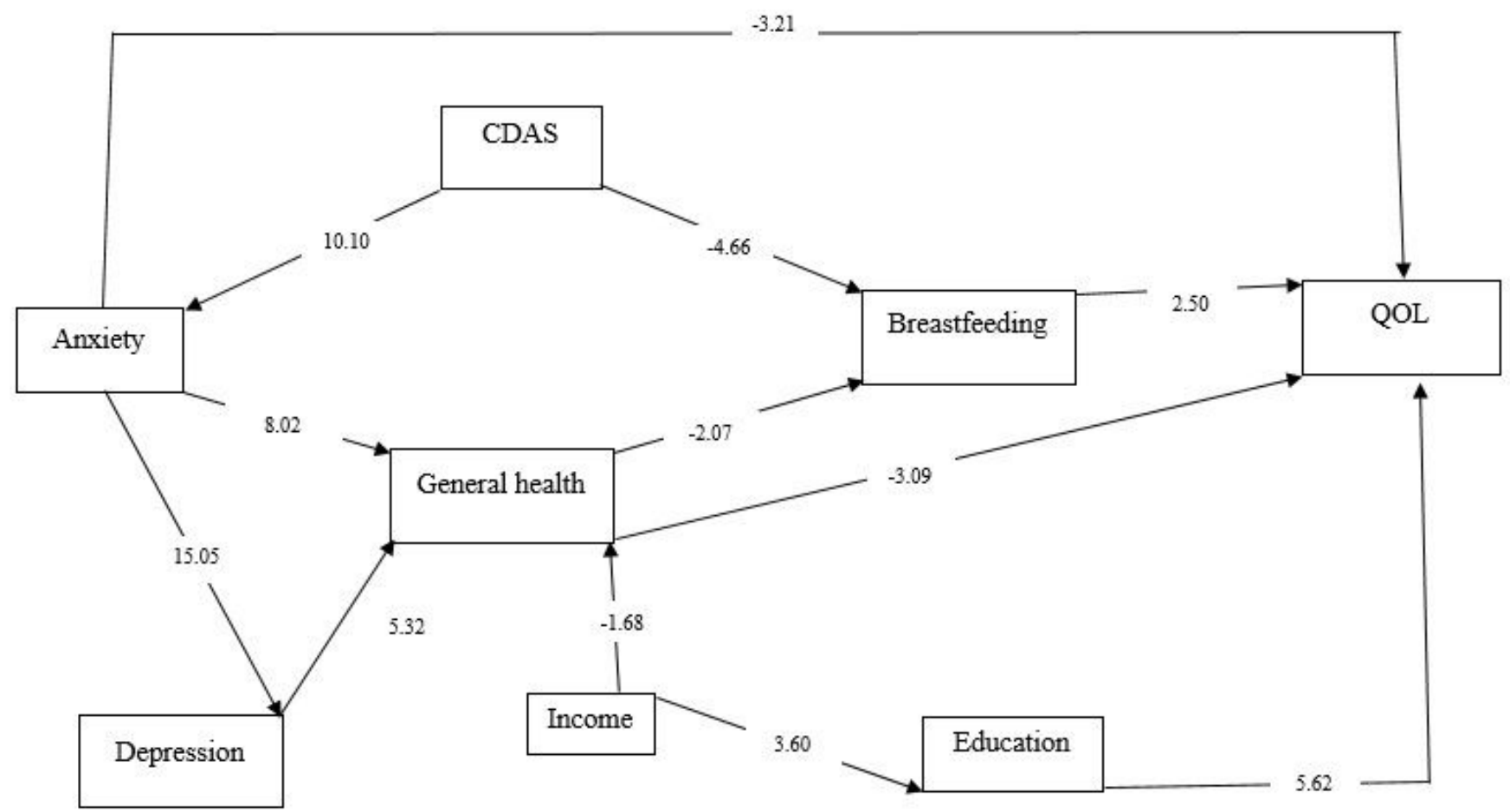

\section{Figure 1}

Path diagram for the predictors of quality of life. 